Richter, Ingo

\title{
Entscheidungsstrukturen für Bildungsfragen in offenen Gesellschaften
}

Zeitschrift für Pädagogik 40 (1994) 2, S. 181-191

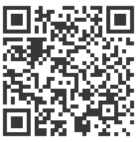

Quellenangabe/ Reference:

Richter, Ingo: Entscheidungsstrukturen für Bildungsfragen in offenen Gesellschaften - In: Zeitschrift für Pädagogik 40 (1994) 2, S. 181-191 - URN: urn:nbn:de:0111-pedocs-108340 - DOI:

10.25656/01:10834

https://nbn-resolving.org/urn:nbn:de:0111-pedocs-108340

https://doi.org/10.25656/01:10834

in Kooperation mit / in cooperation with:

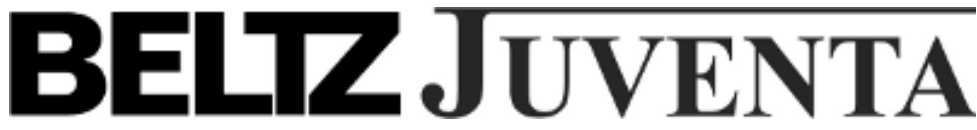

http://www.juventa.de

\section{Nutzungsbedingungen}

Gewährt wird ein nicht exklusives, nicht übertragbares, persönliches und beschränktes Recht auf Nutzung dieses Dokuments. Dieses Dokument ist ausschließlich für den persönlichen, nicht-kommerziellen Gebrauch bestimmt. Die Nutzung stellt keine Übertragung des Eigentumsrechts an diesem Dokument dar und gilt vorbehaltlich der folgenden Einschränkungen: Auf sämtlichen Kopien dieses Dokuments müssen alle Urheberrechtshinweise und sonstigen Hinweise auf gesetzlichen Schutz beibehalten werden. Sie dürfen dieses Dokument nicht in irgendeiner Weise abändern, noch dürfen Sie dieses Dokument für öffentliche oder kommerzielle Zwecke vervielfältigen, öffentlich ausstellen, aufführen, vertreiben oder anderweitig nutzen.

Mit der Verwendung dieses Dokuments erkennen Sie die Nutzungsbedingungen an.

\section{Terms of use}

We grant a non-exclusive, non-transferable, individual and limited right to using this document.

This document is solely intended for your personal, non-commercial use. Use of this document does not include any transfer of property rights and it is conditional to the following limitations: All of the copies of this documents must retain all copyright information and other information regarding legal protection. You are not allowed to alter this document in any way, to copy it for public or commercial purposes, to exhibit the document in public, to perform, distribute or otherwise use the document in public.

By using this particular document, you accept the above-stated conditions of use.

\section{Kontakt / Contact:}

DeDOCS

DIPF | Leibniz-Institut für Bildungsforschung und Bildungsinformation

Informationszentrum (IZ) Bildung

E-Mail: pedocs@dipf.de

Internet: www.pedocs.de

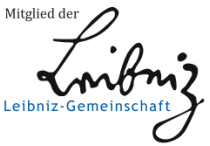




\section{Zeitschrift für Pädagogik}

Jahrgang 40 - Heft 2- März/April 1994

Essay

181 INGO RICHTER

Entscheidungsstrukturen für Bildungsfragen in

offenen Gesellschaften

Thema: Pädagogische Anthropologie

195 JÜrgen OELKERS

Neue Seiten der „Pädagogischen Anthropologie": Einleitung in den

Schwerpunkt

201 DieTer NeumanN

Pädagogische Perspektiven der Humanethologie

229 Johannes Dichgans

Die Plastizität des Nervensystems. Konsequenzen für die Pädagogik

247 ROLF GÖPPEL

Anfänge der menschlichen Subjektivität

265 Franz-E. Weinert/Ulrich Geppert/Jürgen Dörfert/Petra Viek Aufgaben, Ergebnisse und Probleme der Zwillingsforschung dargestellt am Beispiel der Gottschaldtschen Längsschnittstudie

\section{Diskussion}

291 KuRT BeUtLer

Erich Wenigers Militärpädagogik in später Wahrnehmung Eine Zwischenbilanz

303 JOACHIM BENSEL

Ist die Tagesbetreuung in Krippen ein Risiko?

Eine kritische Beurteilung der internationalen Krippenforschung 
329 Peter Dudek

Siegfried Bernfeld: Sämtliche Werke, Bd. 1: Theorien des Jugendalters. Schriften 1914-1938

332 Franz Michael Konrad

Gérard Kahn: Janusz Korczak und die jüdische Erziehung. Janusz Korczaks Pädagogik auf dem Hintergrund seiner jüdischen Herkunft

335 HeINZ RHYN

Gabriele Strobel-Eisele: Schule und soziale Evolution. System- und evolutionstheoretische Untersuchungen zur Entstehung und Entwicklung der Schule

Christel Adick: Die Universalisierung der modernen Schule. Eine theoretische Problemskizze zur Erklärung der weltweiten Verbreitung der modernen Schule in den letzten 200 Jahren mit Fallstudien aus Westafrika

Christel Adick/Uwe Krebs (Hrsg.): Evolution, Erziehung, Schule. Beiträge aus Anthropologie, Entwicklungspsychologie, Humanethologie und Pädagogik

\section{Dokumentation}

341 Pädagogische Neuerscheinungen 
181 INGO RICHTER

Educational Decision making in Open Societies

Topic: Pedagogical Anthropology

195 Jürgen OeLKERS

New Aspects of „Pedagogical Anthropology“ - An Introduction

201 Dieter NeumanN

Pedagogical Perspectives of Human Ethology

229 Johannes Dichgans

The Plasticity of the Neuro-System - Possible consequences for pedagogics

247 ROLF GÖPPEL

The Beginnings of Human Subjectivity

265 Franz-E. Weinert/Ulrich Geppert/Jürgen Dörfert/Petra Viek Tasks, Results, and Problems of Research on Twins Illustrated by Gottschaldt's Longitudinal Study

\section{Discussion}

291 Kurt Beutler

Erich Weniger's Military Pedagogics

In Retrospect - An interim stocktaking

303 JoACHIM BeNSEL

Does Day-Care In Crèches Present a Risk?

A critical assessment of international research on crèches

Reviews

329

Documentation

341 Recent Pedagogical Publications 


\title{
Entscheidungsstrukturen für Bildungsfragen in offenen Gesellschaften
}

\begin{abstract}
Zusammenfassung
Können offene Gesellschaften, die über eine bestimmte Staatsideologie nicht verfügen, sondern unterschiedliche Interessen organisieren, Ziele für ein öffentliches Bildungssystem überhaupt begründen? Nachdem die fraglose Eingliederung des Bildungswesens in die Staatsverwaltung nicht mehr legitim erscheint und nachdem sich eine Organisation nach dem Demokratieprinzip als fragwürdig erwiesen hat, ist zunehmend von vertraglichen Regelungen auch im Bildungswesen die Rede, von Marktmechanismen oder korporativistischen Prinzipien. Privatisierung ist dafür das falsche Stichwort, es scheint eher um die vertragliche Organisation öffentlichen Handelns zu gehen. Die neuere bildungspolitische Entwicklung geht in diese Richtung: De-Institutionalisierung, Effizienzerwartung, Multikulturalität, Wahlfreiheit, Beteiligung.
\end{abstract}

Es war einmal ein König, der ein großes Reich besaß, in dem viele wilde Tiere lebten. Diese Tiere lebten unter sich in Unfrieden und fügten auch dem Gemeinwesen großen Schaden zu. Nachdem der König viele Jahrzehnte lang erfolglos versucht hatte, sie mit Gewalt unter Kontrolle zu halten, wollte er es nun mit Erziehung versuchen. Doch er war des Regierens müde, und so teilte er sein Reich in drei Teile und verfügte, daß jeder seiner drei Söhne zehn Jahre lang versuchen sollte, in je einem der Teile die wilden Tiere zu erziehen. Das gesamte Reich sollte dann derjenige von ihnen erhalten, dem dies am besten gelingen würde.

Der älteste Sohn, der das Gebiet „Germania“ erhielt, wollte die wilden Tiere zu Menschen erziehen und ordnete deshalb ihre Verhältnisse nach dem Vorbild der menschlichen Gesellschaft, denn er meinte, daß allein durch eine Organisation des Tierreichs nach dem Vorbild des Menschenreichs jene wie diese werden würden. Der Löwe wurde also König, das Schaf Polizist, der Esel wurde Lehrer, der Fuchs Bankier usw. Doch nach einigen Jahren stellte sich heraus, daß die wilden Tiere Tiere blieben und nicht wie die Menschen wurden.

Der zweite Sohn, der das Gebiet „Franconia“ regierte, gab den Tieren eine Verfassung, nach der sie alle frei und gleich sein und sich selber regieren sollten, es gab sogar ein Tiergericht, das über die Streitigkeiten nach Maßgabe der Verfassung entscheiden sollte. Der zweite Sohn meinte nämlich, daß die Vernunft sich schon durchsetzen würde, wenn man den Tieren nur die Freiheit schenkte. Doch es änderte sich wenig: Den Raubtieren wurde ihre tägliche Fleischration zugestanden, und die Vögel sangen in völliger Freiheit ihre Lieder unter dem Himmel.

Der dritte Sohn im Gebiet "Anglia" stellte den Tieren ein bestimmtes Gebiet und eine bestimmte Summe Geld zur Verfügung und sagte zu den Tieren, sie sollten gefälligst ihre Anlegenheiten selber regeln; er stellte aber zwei Bedingungen: Sie müßten alles friedlich regeln und dürften ihr Gebiet nicht verlassen und die menschliche Gesellschaft stören. Widrigenfalls würde er ihnen Gebiet und Geld wieder entziehen. Es dauerte entsetzlich lange, bis die Tiere zu Regelungen kamen, die die Bedingungen erfüllten, und es entstand eine gewisse Unübersichtlichkeit, so daß man auch nach zehn Jahren noch nicht genau wußte, wie eigentlich die Verhältnisse in "Anglia“ aussahen. Aber: Es herrschte eine gewisse Ruhe und Ordnung in dem Durcheinander.

Welchem der drei Söhne wird der König das ganze Reich nach Ablauf der zehn Jahre wohl gegeben haben? 


\section{Drei Modelle zu Recht und Erziehung}

Ich werde drei Modelle zum Verhältnis von Recht und Erziehung bilden. Dabei interessieren mich drei Fragen:

1. Folgt die Organisation des Bildungswesens den Organisationsprinzipien der Gesellschaft, oder ist das Bildungswesen ganz anders organisiert als die Gesamtgesellschaft?

2. Soll Organisation erziehen? D.h., steht die Organisation des Bildungswesens selber im Dienste der Erziehungsziele?

3. Hat das Recht eine große oder eine kleine Bedeutung bei der Organisation des Bildungswesens, und entspricht das Recht dem jeweiligen Organisationsmodell?

Ich werde diese drei Modelle kurz vorstellen und mich mit einigen historischen Erscheinungsformen und mit den rechtlichen Ausprägungen beschäftigen.

\section{Das Verwaltungsmodell}

Im Verwaltungsmodell ist das Bildungswesen Teil der Staatsverwaltung. Die Organisationsprinzipien der Staatsverwaltung finden sich auch im Bildungswesen wieder. Durch die Abbildung der gesellschaftlichen Organisationsprinzipien im Bildungswesen sollen die Lernenden frühzeitig mit den Verhältnissen der Gesellschaft vertraut werden, sich in sie einleben, sie mehr und mehr übernehmen. Einer rechtlichen Regelung bedarf es hierzu nicht und schon gar nicht einer juristischen Konfliktregelung. Das Verwaltungsmodell ist juristisch unterdeterminiert.

\section{Das Verfassungsmodell}

Im Verfassungsmodell gehört das Bildungswesen zwar auch in den Bereich der öffentlichen Organisation; es ist jedoch nicht in die Verwaltung integriert, sondern hat einen eigenständigen Ort, der durch Verfassungsprinzipien geregelt wird. Diese Prinzipien entsprechen den Verfassungsprinzipien der Staatsorganisation. Zwar soll dies auch einen erzieherischen Wert haben; doch die erzieherische Kraft einer Verfassung ist problematisch. Die Ansprüche an die rechtliche Regelung sind dagegen deutlich: Die Rechte und Pflichten aller Beteiligten sind gesetzlich geregelt und ebenso die Verfahren der Entscheidungsbildung, und Konflikte werden vor unabhängigen Gerichten ausgetragen und von ihnen gelöst.

\section{Das Vertragsmodell}

Im Vertragsmodell ist die Organisation des Bildungswesens weder durch die Staatsverwaltung noch durch die Staatsverfassung vorgegeben, sondern die Bürger und die gesellschaftlichen Gruppen schaffen sie selber. Die staatlichen Gesetze legen allenfalls bestimmte Bedingungen und Grenzen fest. Das Ver- 
tragsmodell setzt die individuelle Freiheit der Bürger (Wahlfreiheit) und die gesellschaftliche Autonomie der Gruppen (Selbstverwaltung) voraus. Die durch Verträge geschaffene Organisation des Bildungswesens kann gesellschaftlichen Organisationsformen entsprechen, sie muß es aber nicht. Über den erzieherischen Wert solcher Organisationsformen ist wenig bekannt; auch er wird unterschiedlich sein. Der Bedarf an einer allgemeinen gesetzlichen Regelung ist eher gering; die Organisationen schaffen ihr eigenes Recht. Dieses zu schaffende Recht entspricht wiederum den organisatorischen Besonderheiten. Es muß allerdings erst geschaffen werden.

\section{Recht und Erziehung in geschlossenen Gesellschaftssystemen}

Nehmen wir als Beispiel theokratische, nationalsozialistische oder sozialistische Gesellschaften. Ohne sie im übrigen miteinander vergleichen zu wollen, alle drei verbindet die Vorstellung, durch die Organisation des Bildungswesens die Gesellschaft zu formen.

In theokratischen Gesellschaften werden Glauben und Lernen nicht getrennt, der Priester ist der Lehrer, die Kirche ist die Schule, die Bibel ist das Lehrbuch - und dies gilt gleichermaßen für nichtchristliche theokratische Gesellschaften. Eines Schulgesetzes bedarf es nicht, denn der Priester ist gleichzeitig der Richter. Kinder, die in einer solchen Gesellschaft aufwachsen, die andere gesellschaftliche Organisationsformen auch gar nicht kennenlernen, werden - so lautet das Erziehungsziel - andere Gesellschaftsformen auch gar nicht anstreben. Doch diese Erwartung hat getrogen: In Europa hat sich die Säkularisierung in einer jahrhundertelangen Entwicklung durchgesetzt, und zwar auch in denjenigen Ländern, die - wie Deutschland - sehr lange an der religiösen Prägung des Schulwesens festhielten.

Auch der Nationalsozialismus wollte das Bildungswesen nach nationalsozialistischen Prinzipien organisieren: Kämpfen und Lernen sollten nicht getrennt werden; die Schule als Volksgemeinschaft und der Lehrer als Führer, das Parteiprogramm als Gesetz und die Partei als Richter. Doch das Bildungswesen als große, differenzierte herkömmliche und bürokratische Organisation erwies sich als sperrig. Es paßte sich zwar an, und es öffnete sich weit den nationalsozialistischen Ideen. Die nationalsozialistischen Lernenden und Lehrenden wurden gefördert, die Dissidenten unterdrückt. Doch das eigentliche Ziel, die langfristige Prägung der Gesellschaft im nationalsozialistischen Sinne, wurde nicht erreicht. Nach der Niederlage Deutschlands im Zweiten Weltkrieg gab es plötzlich überhaupt keine Nationalsozialisten mehr, und es schien so, als ob es sie nie gegeben hätte.

Ähnliche Beobachtungen lassen sich auch für die sozialistischen Gesellschaften dieses Jahrhunderts machen: Arbeiten und Lernen sollte eins sein; das Bildungssystem sollte den sozialistischen Menschen schaffen. Doch die Integration des Lernens in die Produktion wollte nicht so recht gelingen, die Schule blieb Schule, und ihre Formung nach sozialistischen Prinzipien hielt sich in Grenzen, wenn sie auch durch und durch vom sozialistischen Vokabular geprägt wurde und die Partei zu bestimmen hatte. Der sozialistische Mensch jedenfalls ist auch in 70 Jahren nicht geschaffen worden. 
Ich nenne diese Organisationsform des Bildungssystems „Verwaltungsmodell", obwohl der Ausdruck "Ideologiemodell" vielleicht treffender wäre, denn die Prägung durch eine gesellschaftspolitische Idee, der Erziehung und Recht untergeordnet werden, ist für dieses Modell typisch. Man muß jedoch die Frage stellen, ob dieses Modell mit dem Verschwinden der ideologischen Leitideen in Westeuropa ebenfalls verschwunden ist. Dies scheint nämlich nicht der Fall zu sein, denn verfassungs- oder vertragsmäßige Organisationsformen haben sich nicht überall und vollständig durchgesetzt.

Halten wir also für das „Verwaltungsmodell“ einstweilen fest:

1. Institutionalisierte Bildung ist ein Staatsmonopol.

2. Es gibt eine zentrale Leitung, die durch die Regierung ausgeübt wird.

3. Die Lehrenden sind Staatsdiener mit einer mehr oder weniger starken professionellen Prägung.

4. Die Lernziele werden durch die Regierung bestimmt; sie können einer einheitlichen Staats- und Erziehungsideologie entspringen, sie können aber auch den pragmatischen Bedürfnissen jeweiliger Mehrheiten entsprechen.

5. Die staatlichen Lernziele werden durch die weisungsabhängigen Staatsdiener in den Bildungsinstitutionen durchgesetzt; gesetzlicher Regelungen repräsentativer Körperschaften bedarf es nicht; die Lernenden und die Lehrenden können sich auf Individualrechte im Bildungswesen nicht berufen; eine gerichtliche Kontrolle findet nicht statt.

\section{Freiheit und. Gleichheit in einem rechtsstaatlich verfaßten Bildungssystem}

Art. 1 der französischen Erklärung der Menschen- und Bürgerrechte von 1789 stellte fest, daß die Menschen vor dem Gesetz frei und gleich geboren seien und es blieben und daß soziale Unterschiede nur auf das Gemeinwohl gegründet werden könnten, und Titel I der Verfassung von 1791 versprach den Franzosen ein allgemeines, kostenloses öffentliches Unterrichtswesen. Ein solches Unterrichtswesen schloß jede partikulare Prägung durch religiöse oder politische Interessen aus; es sollte allein unter dem allgemeinen Gesetz stehen, das Ausdruck der „volonté générale" ist.

Ein solches Bildungswesen folgt dem „Verfassungsmodell“. Freiheit und Gleichheit im Rahmen der für alle geltenden Gesetze sind die Prinzipien der Staatsorganisation, sie sollen auch für das Bildungswesen gelten. Dies muß notwendig öffentlich sein, denn nur ein öffentliches und kostenloses Bildungswesen kann die gleiche Freiheit aller Bürger gewährleisten; private Bildungseinrichtungen dienen partikularen Interessen und sind daher nicht zuzulassen. Dies bedeutet vor allem eine deutliche Trennung von Kirche und Staat. Diese Verfassung des Bildungssystems bedarf der gesetzlichen Regelung, denn die Freiheit der Bürger darf nur durch Gesetze eingeschränkt werden, und die Gleichheit der Bürger muß durch Gesetze konkretisiert werden. Über die Gewährleistung der Freiheit und die Durchsetzung der Gleichheit wachen die Gerichte, die nur dem Gesetz verpflichtet sind. Doch welchen Zielen diente 
diese sehr abstrakte Organisationsstruktur des Bildungssystems? Zur Zeit der Französischen Revolution sollte sie sicher den Zielen der Revolution dienen, der Schaffung der Gesellschaft der Freien und Gleichen. „Wenn man nicht von Kindheit an lernt, republikanisch oder monarchisch, katholisch oder atheistisch zu sein, wird der Staat nie Nation werden", sagte Napoleon bald darauf. Auch die republikanische Bildungsverfassung stand also im Dienst der republikanischen Staatsform - ebenso wie in den theokratischen, monarchischen oder anderen geschlossenen Systemen.

Doch das französische Modell eines konstitutionellen Bildungssystems setzte sich trotz des fortwirkenden Pathos der Französischen Revolution selbst im eigenen Land nicht durch. Die Freiheit wurde bald nicht allein als Freiheit, das öffentliche Bildungssystem besuchen zu dürfen, verstanden, sondern auch als Freiheit, freie Schulen i. S. von staatsfreien, meist kirchlichen Schulen zu gründen, zu unterhalten und zu besuchen. „L'enseignement libre“ wurde - in einer Verkehrung des revolutionären Freiheitsbegriffes - das Schlagwort im Kampf für die religiösen Schulen, die im 19. Jahrhundert nach und nach staatlich genehmigt und schließlich seit der Mitte des 20. Jahrhunderts auch staatlich finanziert wurden. Aus der Gleichheit aller wurde in einem äußerst differenzierten Bildungssystem sehr schnell eine Chancengleichheit zu Beginn und eine äußerst starke Ergebnisungleichheit am Ende der Bildungsbiographien. Das Gesetz, das Freiheit und Gleichheit doch nur nach Maßgabe des Gemeinwohls einschränken sollte, regelte schließlich ein duales System staatlicher und privater Schulen und ein Bildungssystem, das die gesellschaftliche Ungleichheit aufgrund einer scheinbaren Gleichheit im Bildungssystem nur um so besser reproduzierte (BouRdiEU/PASSERON 1971). Von einer gerichtlichen Korrektur dieser Ergebnisse aufgrund von verfassungsrechtlichen Freiheits- und Gleichheitsrechten konnte in der französischen Entwicklung überhaupt keine Rede sein.

Doch die Entwicklung des französischen Bildungssystems sollte uns nicht daran hindern, das Verfassungsmodell des Bildungswesens in seiner ursprünglichen Radikalität noch einmal nachzuzeichnen:

1. Institutionalisierte Bildung ist ein Staatsmonopol, private Schulen sind verboten; es besteht eine strikte Trennung von Staat und Kirche; ein Religionsunterricht findet in der Schule nicht statt.

2. Alle Kinder bzw. ihre Eltern haben ein gleiches Recht auf Bildung nach Maßgabe von Interesse und Qualifikation; es besteht Chancengleichheit auf allen Ebenen.

3. Die Lehrenden sind Staatsdiener mit einer mehr oder weniger starken professionellen Prägung.

4. Die Schulorganisation, die Anstellung der Lehrer und die Lehrpläne beruhen auf dem Prinzip der demokratischen Wahl, an der alle Staatsbürger zu beteiligen sind.

5. Das Gesetz regelt Rechte und Pflichten aller Beteiligten einschließlich der Wahlverfahren; alle Entscheidungen unterliegen der gerichtlichen Kontrolle.

Es ist verblüffend, daß das Verfassungsmodell des Bildungssystems, das mit seinen Elementen der Öffentlichkeit, Repräsentation, Gesetzlichkeit, Grund- 
rechtsgewährung und Gerichtskontrolle doch in so hohem Maße den Staatsverfassungen der westlichen Industrienationen entspricht, sich im Bildungssystem so wenig durchgesetzt hat.

\section{Markt oder Korporationen}

Das Vertragsmodell kann in zwei völlig unterschiedlichen Formen auftreten, und zwar

1. als Marktmodell, wenn private Anbieter auf einem Markt für Bildung privaten Nachfragern die Dienstleistungen des Bildungswesens anbieten und wenn eine freie Preisbildung grundsätzlich zulässig ist, und

2. als Korporationsmodell, wenn öffentliche Organisationen mit dem Staat und untereinander die Bedingungen aushandeln, zu denen sie die Dienstleistungen des Bildungswesens den Bürgern anbieten.

Beiden Modellen ist gemeinsam, daß die Bürger die Wahl zwischen verschiedenen Angeboten haben. Der Unterschied besteht darin, daß es sich im ersten Fall um eine privatwirtschaftliche Organisationsform handelt, daß im ersten Fall die Konditionen frei ausgehandelt werden, während sie im zweiten Fall bestimmten Beschränkungen unterworfen sind. Beide Modelle können auch in einem gemischtwirtschaftlichen Modell vereint werden.

Das Marktmodell ist im Bereich der institutionalisierten Bildung großen Beschränkungen unterworfen, denn Schule und Hochschule sind in Westeuropa grundsätzlich staatlich. Soweit private Schulen und Hochschulen überhaupt vorgesehen sind, müssen sie bestimmte Zulassungsvoraussetzungen erfüllen, die sie zu einem Annex des staatlichen Schulwesens machen, und da sie in ihrer Preisgestaltung frei sind, hängen sie alle mehr oder weniger von staatlichen Subventionen und/oder privaten Spenden ab. Das ist kein privatwirtschaftlicher Markt, sondern ein öffentlich geregeltes Verwaltungssystem mit gewissen Spielräumen. Anders ist es jedoch im Bereich der beruflichen Bildung, soweit sie in Betrieben und nicht in Schulen organisiert ist, und ebenso in der Weiterbildung, wo es einen Markt freier Anbieter gibt. Hier hat sich also das Marktmodell weitgehend durchsetzen können, wenn es auch bestimmte staatliche Reglementierungen bei der Zulassung und der Preisgestaltung gibt. In der Gestaltung der Berufsausbildung und Weiterbildung nach dem Marktmodell spiegelt sich ihre pädagogische Konzeption, die von einer Integration der Ausbildung und Weiterbildung in die Betriebe ausgeht. So ist es nur folgerichtig, daß Berufsausbildung und Weiterbildung ebenso wie Produktion und Verteilung von Waren und Dienstleistungen organisiert werden. Es ist auch immer wieder behauptet worden, daß mit dieser Organisationsform auch ein ganz bestimmtes Erziehungsziel verbunden wird, die Eingliederung in kapitalistische Produktionsprozesse einschließlich der Übernahme der entsprechenden Werte und Normen (CARNOY 1972). Deutlich ist jedenfalls, daß mit der Organisation von Berufsausbildung und Weiterbildung nach privatwirtschaftlichen Grundsätzen die Übernahme der Rechtsgrundsätze des Wirtschafts- und Arbeitsrechts einhergeht: Vertragsfreiheit und Tarifautonomie in den Grenzen einer staatlichen Aufsicht. 
Wirft man einen Blick auf Formen nichtinstitutionalisierter Bildung, wie z.B. die Buchproduktion und -verteilung, oder auf die Presse, so dominieren marktwirtschaftliche Modelle, im Bereich der sonstigen Massenkommunikationsmittel gemischtwirtschaftliche Modelle - doch hiervon soll in diesem Rahmen nicht mehr die Rede sein.

Für ,reine Korporationsmodelle“ gibt es weder in Westeuropa noch sonst irgendwo auf der Welt Beispiele; es handelt sich immer um Mischformen zwischen verschiedenen Modellen. Am nächsten kommt dem Korporationsmodell das niederländische Bildungssystem mit seinen vielgestaltigen Angeboten (LIKET 1993). In den Niederlanden hat sich aus den Auseinandersetzungen über die religiöse Prägung des Schulwesens ein Vier-Säulen-Modell des Schulwesens entwickelt, in dem neben einem öffentlichen Schulwesen, das nur etwa 30 Prozent der Schüler umfaßt, ein katholischer und evangelischer Sektor sowie ein weltlicher Bereich stehen, in dem Schulen unterschiedlichster Prägung zusammengefaßt sind. Das Besondere der niederländischen Organisationsform ist nun, daß im Prinzip jede gesellschaftliche Gruppe ein eigenes Schulwesen unterhalten kann (Art. 23 der Verfassung), in dem sie über Gründung und Schließung von Schulen, über Beschäftigung von Lehrern und Leitern, den Zugang und Abgang von Schülern sowie über das Curriculum entscheidet allerdings unter der Aufsicht der staatlichen Schulbehörden. Dieses Bildungssystem ist in gewisser Weise ein parzelliertes „Verwaltungsmodell“, wie ich es oben gekennzeichnet habe, nur daß nicht eine herrschende Ideologie das System dominiert, sondern daß es jede gesellschaftliche Gruppe auf ihre eigene Art und Weise tun kann. Das System entspricht deshalb in besonderem Maße den Strukturprinzipien der pluralistischen Gesellschaft. Da es jedoch auch einen Sektor der öffentlichen Schulen gibt, handelt es sich auch hier um ein "gemischtes System".

In allen westeuropäischen Bildungssystemen finden sich korporationsrechtliche Elemente, d.h., daß das Bildungssystem nicht allein auf dem Gegensatz zwischen der staatlichen und der privaten Sphäre beruht, auf der Unterscheidung von staatlichen und privaten Schulen. Es lassen sich die folgenden korporationsrechtlichen Elemente feststellen:

1. Die sogenannten Privatschulen werden in der Regel nicht von privaten Unternehmern oder von interessierten Bürgern bzw. Eltern unterhalten, sondern von den Kirchen, die in gewisser Weise auch öffentliche Korporationen sind.

2. In vielen westeuropäischen Ländern haben die Kommunen großen Einfluß auf die Schulen, und zwar insbesondere auf die Standorte, den Bau und die Unterhaltung sowie z.T. auch auf das Personal, und die Kommunen sind ebenfalls besondere öffentliche Korporationen.

3. Einige Länder räumen den Arbeitergeber- und Arbeitnehmerverbänden Einfluß auf die Berufsausbildung ein, einige Länder sogar auf das allgemeinbildende Schulwesen, das sie vertraglich regeln können, wodurch auch eine korporationsähnliche Lösung entsteht.

4. Es kommt hinzu, daß - insbesondere im Bereich der Hochschulen - manche Bildungsinstitutionen selber Korporationen sind, denken wir nur an die ehrwürdige Geschichte von Oxford und Cambridge. 
Es versteht sich, daß das „gemischte“ System einen hohen Bedarf an rechtlichen Regelungen hat, und zwar sowohl gesetzlicher wie vertraglicher Art. Eine besondere Erziehungsfunktion wird man diesem Bildungssystem nicht zusprechen können; in ihm kommt vielmehr der für das Bildungssystem typische Interessenkonflikt zum Ausdruck.

\section{Bildungsverfassungen in Westeuropa}

Wenn man die derzeitigen Bildungssysteme Westeuropas betrachtet, so läßt sich nicht sagen, daß sie alle oder auch nur einzelne einem bestimmten der drei gekennzeichneten Modelle folgen, sondern in allen Ländern haben sich im Laufe der historischen Entwicklung verschiedene Modellelemente überlagert. Vielleicht lassen sich drei Gruppen von Ländern bilden:

A. Frankreich und die Länder des romanischen Rechtskreises wird man noch am ehesten dem „Verwaltungsmodell“ zuordnen können (HALls 1976). Die verfassungsrechtliche Prägung ist - sieht man von der Privatschulfreiheit $a b$ - schwach; gesetzliche Regelungen konkretisieren nicht Grundrechte und gewähren keine Verfahrensrechte, sondern legalisieren im Grunde nur administrative Regelungen. Die gerichtliche Kontrolle ist nur schwach ausgeprägt.

B. Deutschland, Osterreich und die Schweiz könnte man noch am ehesten dem „Verfassungsmodell" zuordnen. Es bestehen umfangreiche gesetzliche Regelungen des Bildungswesens, begleitet von einer weitreichenden gerichtlichen Kontrolle bildungspolitischer und pädagogischer Entscheidungen. Bildung wird als Ausübung eines Grundrechts verstanden, wenn auch das Gleichheitspostulat nicht stärker verwirktlicht ist als in anderen Ländern. Die Bildungseinrichtungen besitzen Selbstverwaltungsgremien, die gewählt werden. Zu diesen Elementen des Verfassungsmodells kommen jedoch korporative Elemente hinzu, und zwar durch die starke Stellung der Kirchen und Kommunen, durch private Schulträger und gewisse marktwirtschaftliche Elemente durch die Organisation der Berufsausbildung.

C. In Skandinavien, in den Niederlanden und Großbritannien findet man gemischte Bildungsverfassungen, jedenfalls scheinen hier Elemente des "Vertragsmodells" eine größere Rolle zu spielen als in anderen Ländern. Großbritannien fällt durch die starke Stellung der Kommunen auf, die jedoch neuerdings durch marktwirtschaftliche und korporativistische Elemente relativiert wird (MANN 1979); jedenfalls ist die Ausprägung des Verwaltungs- wie des Verfassungsmodells gering. Skandinavien ist durch die Kooperation von Arbeitgeber- und Arbeitnehmerorganisationen stark korporativistisch geprägt; doch bestehen starke Elemente des Verfassungsmodells fort (WrLLMANN 1980). Die Niederlande wurden bereits als stark korporativistisch gekennzeichnet.

Doch diese Gruppierungen können nur mit großem Vorbehalt vorgenommen werden; neuere Entwicklungen könnten sie sowieso obsolet werden lassen. 


\section{Bildungsverfassungen in der Zukunft}

Die Zukunft wird m. E. eine Abkehr von den drei Annahmen bringen, die ich der Modellbildung zugrunde gelegt habe:

1. Die Sozialisationskraft von Institutionen wird abnehmen; da Bildung nicht in einer Institution allein erfolgt, sondern in einer Vielzahl von Verfahren und Einrichtungen, werden wir nicht mehr auf die prägende Kraft einer einzigen Einrichtung vertrauen (HENTIG 1993).

2. Die Organisation von Bildungsinstitutionen folgt nicht mehr dem Modell der Staatsorganisation; Schulen und Universitäten sind nicht Körperschaften wie der Staat, sondern Körperschaften eigener Art (FAUSER 1986).

3. Die Grenzen der rechtsstaatlichen Verfassung des Bildungswesens scheinen erreicht; Gesetzgebung und Rechtsprechung werden im Bildungswesen der Zukunft vermutlich eher eine geringere Rolle spielen als in einigen Ländern in der unmittelbaren Vergangenheit (STAUPE 1986).

Die Zukunft könnte möglicherweise eine Hinwendung zum „Vertragsmodell“ bringen. Mit dem Verschwinden einheitlicher Ideologien und mit der zunehmenden Differenzierung der gesellschaftlichen Ansprüche an das Bildungswesen verliert das "Verwaltungsmodell" zunehmend seine Legitimation. Das „Verfassungsmodell" hat sich mit seinen egalitären und demokratischen Ansprüchen nicht durchgesetzt, seine rechtsstaatlichen Ausprägungen scheinen an Grenzen gestoßen zu sein, und seine freiheitlichen Aspekte begründen eine Pluralisierung, die dem unitaristischen und zentralistischen Ansatz des Verfassungsmodells widerstrebt. So stellt sich die Frage, ob denn das „Vertragsmodell" mit seiner marktwirtschaftlichen und korporativistischen Struktur die derzeitigen bildungspolitischen Probleme Westeuropas lösen kann.

Ich möchte fünf neuere bildungspolitische Tendenzen erwähnen und fragen, ob das "Vertragsmodell" zu ihrer Bearbeitung beitragen kann:

\section{De-Institutionalisierung der Bildung}

Es spricht einiges dafür, daß neben die in Schulen und Hochschulen institutionalisierten Bildungsprozesse zunehmend informelle Formen der Bildung treten, die stark durch die neuen Kommunikationsmittel bestimmt sein werden. Hier werden eindeutig marktwirtschaftliche und korporativistische Organisationsformen vorherrschen (FAURE 1973).

\section{Effizienzerwartungen}

Die Beteiligten, die Abnehmer der im Bildungswesen produzierten Qualifikationen und der von Steuern lebende Staat, werden zunehmend nach der Kosten-Nutzen-Relation der institutionalisierten Bildung fragen. Dies wird den öffentlichen Druck in Richtung auf die Einfügung marktwirtschaftlicher Elemente in das öffentliche Bildungswesen verstärken und zur Einführung von Verfahren der Rechenschaftslegung führen, die die korporativistischen Tendenzen stärken wird (KienBaum 1991). 


\section{Multikulturalität}

Die Migrationsbewegungen der Dekolonisierung der Welt, der ökonomischen Wachstumsperioden und der zukünftigen europäischen Integration haben bzw. werden multikulturelle Gesellschaften entstehen lassen, die zunehmend pluralistische Strukturen des Bildungswesens aufweisen werden. Durch einen bloßen kulturellen Minderheitenschutz im Rahmen des „Verfassungsmodells“ wird sich diese Entwicklung nicht einfangen lassen. Nur die Gewährung kultureller Autonomie entspricht der entstehenden Lage, und dies bedeutet: Regelungen nach dem korporativistischen Vertragsmodell (SCHULTE 1990).

\section{Wahlfreiheit}

Weder das Verwaltungs- noch das Verfassungsmodell bieten den Lernenden und ihren Eltern ein großes Maß an Wahlfreiheit; beide gehen im Grunde davon aus, daß individuelle Präferenzen angesichts des Prinzips einer einheitlichen Ideologie bzw. der gleichen Freiheit aller keine Rolle spielen dürfen. Der Trend nach mehr Wahlfreiheit ist jedoch deutlich, und nur durch die marktwirtschaftlichen und/oder die korporativistischen Elemente des Vertragsmodells kann dem entsprochen werden (CoONs/SUgARMAN 1978).

\section{Beteiligung}

Über die Wahlfreiheit, d.h. die Auswahl aus einem bestehenden Angebot, hinaus zeichnet sich aber ein deutliches Interesse vieler Bürger und ihrer Organisationen an einer Beteiligung an der Gestaltung des Angebots selber ab. Die europäischen Versuche zu einer solchen Beteiligung im Rahmen des Verfassungsmodells sind nicht sehr überzeugend ausgefallen; neue, weiter gehende Versuche in dieser Richtung scheinen mir auf der Tagesordnung zu stehen. Auch sie können nur in Richtung des ,Vertragsmodells" gehen (RdJB 1987, Heft 3, und 1990, Heft 4).

\section{Märchen können wahr werden}

Als nun die zehn Jahre vorüber waren, bestellte der König seine drei Söhne zu sich an scin Bett, denn er war mittlerweile alt und schwach geworden, und sagte: „Das Reich soll mein jüngster Sohn erhalten, denn er hat meine Bedingungen erfüllt. Die wilden Tiere verlassen das ihnen zugewiesene Gebiet nicht mehr, und sie wahren Ruhe und Ordnung, und das für sie ausgegebene Geld reicht aus.“ Zu dem ersten Sohn sagte er: „Du hast die Kraft der Erziehung überschätzt und bist deshalb gescheitert“, und zu dem zweiten Sohn sagte er: „Du hast die Kraft der Verfassung überschätzt; Verfassungen setzen den Status quo fort." Da sagten die beiden älteren Söhne einstimmig: „Wie kannst du dich für ein System entscheiden, das Du gar nicht kennst?“ „Der Erfolg wird letztlich darüber entscheiden", meinte der König und starb. 


\title{
Literatur
}

Bourdieu, P./Passeron, J.-Cl.: Die Illusion der Chancengleichheit. Stuttgart 1971.

CARnoY, M.: Schooling in a Corporate Society. New York 1972.

Coons, J. E./Sugarman, S. D.: Education by Choice. Berkeley/Los Angeles/London 1978.

FAure, E.u.a.: Wie wir leben lernen. Der UNESCO-Bericht über Ziele und Zukunft unserer Erziehungsprogramme. Reinbek 1973.

Fauser, P.: Pädagogische Frciheit in Schule und Recht. Oxford/New York/Frankfurt a, M. 1986.

Halls, W.D.: Education, Culture and Politics in Modern France. Oxford/New York/Frankfurt 1976.

Hentig, H. von: Die Schule neu denken. München/Wien 1993.

KIENBAUM Unternehmensberatung (Hrsg.): Organisationsuntersuchung im Schulbereich. Frechen 1991.

LiKeT, T.M.: Freiheit und Verantwortung. Gütersloh 1993.

MANN, J.F.: Education. London 1979.

RdJB: Recht der Jugend und des Bildungswesens: Beiträge zu Schulverwaltung und Schulverfassung Heft 3, 1987, Heft 4, 1990.

Schulte, A.: Multikulturelle Gesellschaft: Chance, Ideologie oder Bedrohung. In: Aus Politik und Zeitgeschichte B23-24 (1990), S. 3-15.

STAuPE, J.: Parlamentsvorbehalt und Delegationsbefugnis. Berlin 1986.

WILlmaNN, B.: Bildungswesen, Chancengleichheit und Beschäftigungssystem. Vergleichende Daten und Analysen zur Bildungspolitik in Schweden. München 1980.

\begin{abstract}
Open societies which lack official ideologies but try to organize conflicting interests, can they base their educational system on educational goals at all? The traditional integration of the educational system into the state administration is no longer acceptable. To organize education just like the state on the basis of democratic principles is highly questionable. So, more and more contract regulation seems to be adequate, market principles or corporate structures. But, private business is not the model. Public contractual regulation seems instead to be the keyword. New trends in educational policy point into this direction: de-institutionalization of the educational system, efficiency expectations, multiculturalism, choice, participation.
\end{abstract}

Prof. Dr. Ingo Richter, Deutsches Jugendinstitut, Freibadstr. 30, 81543 München 